\title{
El cuerpo puesto al debate: bosquejo sobre el establecimiento de las problemáticas trans en el contexto chileno
}

\section{Body put to debate: outline on the establishment of trans problems in the Chilean context}

Carlos Jaque Paéz*

\section{Resumen}

El presente artículo refiere sobre la introducción de las llamadas "problemáticas trans" (aquellas que conciernen de manera general a identidades travesti, transgénero y transexuales) en el ámbito del debate público en el contexto chileno. Bajo esta premisa, se realiza primero un recorrido por distintas figuras identitarias trans en el desarrollo histórico occidental del último siglo, para posteriormente observar su inmersión en el contexto local. Para ello se observa el abordaje realizado a propósito de las problemáticas trans en distintos tópicos, en particular a través del trato dado por los medios de comunicación, la preocupación desde el análisis de Derechos Humanos, el trabajo comunitario y legislativo realizado por organizaciones sociales, y la paulatina importancia que han cobrado dichas problemáticas en los estudios académicos. Finalmente, se reflexiona sobre la relevancia del cuerpo puesto al debate público, permitida gracias a la introducción de las problemáticas trans.

Palabras clave: Sexo/género, problemáticas trans, identidad de género, Sociología del Cuerpo, Ley de Identidad de Género.

\begin{abstract}
This article refers to the introduction of the so-called "trans issues" (those that generally concern transvestite, transgender and transsexual identities) in the context of public debate in the Chilean context. Under this premise, a review through different trans identity figures in the Western historical development of the last century is carried out, to later observe their immersion in the local context. To this end, the approach taken on trans problems in different topics is observed, particularly through the treatment given by the
\end{abstract}

‘Sociólogo de la Universidad de Chile. carlos.jaque@ug.uchile.cl 
media, the concern from the analysis of Human Rights, community and legislative work carried out by social organizations, and the gradual importance that these problems have gained in academic studies. Finally, it reflects on the relevance of the body to the public debate, allowed thanks to the introduction of trans issues.

Key words: Sex/gender, trans issues, gender identity, Sociology of the Body, Gender Identity Law.

Fecha de recepción: Abril 2019

Fecha de aprobación: Diciembre 2019

\section{Introducción ${ }^{1}$}

La discusión a propósito de las identidades trans en el ámbito de lo público puede ser considerada relativamente reciente en el contexto nacional, teniendo presente que solamente hacia el año 2013 ingresa al Congreso el proyecto para una Ley de Identidad de Género (LIG), cuya discusión legislativa hasta su aprobación a finales de 2018 fue una de las principales premisas que permitieron el acceso al debate social en torno a las llamadas "problemáticas trans" ${ }^{2}$. El objetivo de dicho proyecto fue la tramitación de una ley que permitiese "la rectificación de la partida de nacimiento respecto al nombre y sexo, atendiendo al género con el que el solicitante se siente identificado" (Álvarez, del Pino y Vial, 2015: 374); lo que indica que se buscó con ello una mayor preponderancia de la identidad de género por sobre el sexo para la autodefinición de los sujetos.

Si tomamos en cuenta que para la elaboración de este proyecto (del Pino y Verbal, 2015) se considera la clásica distinción entre género y sexo (donde mientras el primero hace referencia a factores socio-culturales de significación, el segundo evocaría factores biológicos), la LIG aprobada se abre como una oportunidad de considerar con mayor relevancia lo que el imaginario social puede decir acerca de los propios cuerpos y, en mayor medida, acerca de los cuerpos que se tienden a agrupar tras el concepto "trans" (en general, individuos transexuales, transgénero, travesti $y$ en ocasiones a intersexuales).

1 El presente artículo corresponde a una versión resumida y adaptada del trabajo investigativo "Procesos de (re)significación sexual del cuerpo en usuarios trans de OTD Chile", específicamente, de los apartados destinados a la recolección de antecedentes y al planteamiento del problema de investigación, presentado en el segundo semestre de 2018 como memoria para optar al título de Sociólogo en la Facultad de Ciencias Sociales de la Universidad de Chile.

2 Por "problemáticas trans" se apunta a aquellos conflictos concernientes a las identidades trans en tanto se exponen a un contexto social regido por dictámenes heteronormados y ante una premisa político-sexual binaria. 
Esta apertura al debate en torno a las identidades trans en el contexto chileno se expresa no sólo en la disputa por una LIG, sino también se manifiesta en diversos ámbitos tales como el surgimiento y consolidación de distintas agrupaciones y comunidades de apoyo a las identidades trans, su reciente cobertura en artículos de prensa y reportajes periodísticos, la realización de documentales respecto a la situación en el país, el creciente interés académico y jurídico, y la apertura de estas temáticas a través de distintas expresiones culturales, entre otras. Dentro de este proceso de visibilización y disputa, gran importancia tiene también la constante homologación de estas exigencias con las de otros sectores de la diversidad sexual debatidas en los últimos años (leyes antidiscriminación, matrimonio igualitario, etc.).

No obstante a esto último, podemos considerar que en este caso particular, a diferencia de otras disputas llevadas a cabo por parte de los movimientos de la diversidad sexual, son justamente los mismos cuerpos quienes se encuentran en debate de manera casi explícita al estar continuamente expuestos ante los ojos de la sociedad y del Estado. Aún más, estamos frente al proceso de cómo se significa el cuerpo en tanto que tal, dando luz sobre el eje primordial de dicha disputa: otorgar mayor relevancia a la identidad de género implica devaluar el procedimiento tradicional por el que se asigna el sexo biológico y por el cual se suponen representaciones corporales e identitarias específicas. Entonces, reflexionar sobre el sexo y el género supone también repensar cuáles son las formas en que el cuerpo adquiere su significado.

La búsqueda por concebir a los sujetos trans como individuos "de derechos" por parte de las organizaciones del movimiento por la diversidad, va más allá de implicancias solo jurídicas o de reivindicación identitaria (cuestiones que se manifiestan de un modo más explícito), sino que también evidencia la elaboración y emergencia de nuevos discursos sociales que buscan la (re)significación de sus cuerpos, los cuales se han encontrado históricamente estigmatizados en la sociedad chilena. En esta búsqueda, el cuerpo trans puede entenderse como un espacio de disputa, pues para otorgar validez a las identidades trans en el imaginario social, será necesario elaborar discursos que otorguen nuevos sentidos a dichos cuerpos.

\section{Origen e historización de las identidades trans}

Este apartado comenzará por mencionar la definición otorgada en 1966 por el endocrinólogo Harry Benjamin al término transexual, y que significó la entrada de una nueva categoría que permitiría al discurso médico comprender la posición de aquellos individuos que no se ajustaban a la clásica significación dicotómica del sexo. Según Benjamin, la principal característica del transexual es su "profunda infelicidad como miembro del sexo (o género) que a él o ella se le ha asignado según la estructura anatómica del cuerpo, particularmente los genitales" (1999: 11). En otras palabras, en el transexual existiría un "malestar" específico, que lo haría escapar de los márgenes en los cuales se comprende tradicionalmente la división sexual, por lo que la utilización del prefijo trans otorga sentido a dichos individuos, en tanto los ubica "más allá" del sexo. 
El trabajo de difusión realizado por Benjamin estableció los parámetros medicalizados por los cuales se entendería socialmente la transexualidad desde mediados del siglo $\mathrm{XX}$ a la actualidad, y también permitiría establecer el nexo para su patologización por medio del diagnóstico de disforia de género, el cual se mantiene en versiones actuales de manuales psiquiátricos, como el DSM ${ }^{3}$ (Noseda, 2012). Aún más, en algunas ocasiones, el diagnóstico de dicha patología se convertirá en requisito para poder optar al procedimiento quirúrgico que establece la "veracidad" del transexual. Sería el mismo Benjamin (1999: 15-16) quien establecería, por medio de una escala graduada, que el transexual se vuelve "verdadero" a través de la llamada Cirugía de Reasignación Sexual (CRS).

El origen médico del concepto transexual no es fortuito, pues responde a un proceso de refinamiento de las técnicas de producción de saber en torno al propio cuerpo, el cual "surge como un campo de intervención en el que, precisamente, cuerpo, conocimiento y población serán materia de importancia fundamental para las operaciones políticas" (Fernández, 2004: 19). Estas formas de intervención discursiva sobre el cuerpo son descritas por Michel Foucault como una vinculación entre un orden político y un orden corporal que permitiría la incorporación de "sexualidades periféricas" (1987: 56) en sus discursos con el afán de su especificación. Así, la categoría de Benjamin es utilizada hasta el día de hoy para diferenciar al transexual del travesti, diferenciación que se arraiga en la experiencia corporal del sujeto (Benjamin, 1999: 14): mientras en el transexual los órganos genitales son fuente de "malestar", en el travesti estos mismos órganos son fuente de deseo.

Respecto a esta diferenciación, Dave King (1998) establece una cronología del concepto travestismo segmentada en cuatro fases, que se corresponden a su vez con cuatro momentos histórico-políticos: (1) entre 1870 y 1920, tras un extenso periodo de criminalización, se produce y recopila numerosa información sobre casos de hombres y mujeres que se interesan por adscribirse al rol del sexo opuesto; (2) entre 1920 y 1950, el concepto ya se encuentra establecido y utilizado por la literatura académica, y se comienzan a practicar las primeras cirugías de reasignación sexual; (3) entre 1950 y 1965, surge el interés académico-político por las hasta entonces denominadas "aberraciones sexuales" (entre las cuales se encuentra el travestismo), y que permitiría el ingreso del concepto "género" en su discurso; y (4) desde 1965 hasta los 80', decae el interés médico por el travestismo tras la categorización llevada a cabo por Benjamin, y la figura del travesti adquiere un rol más bien de reivindicación política de formas sexuales estigmatizadas. Si bien la distinción entre travestismo y transexualidad parece ser

${ }^{3}$ EI DSM ("Manual Diagnóstico y Estadístico de Enfermedades Mentales") es una guía desarrollada en distintas ediciones desde mediados del siglo pasado por la Asociación Estadounidense de Psiquiatría (APA), y que sirve como referencia para la definición y diagnóstico de enfermedades mentales. Hasta su edición número IV, se conservaba el diagnóstico de "Trastorno de identidad de género"; para finalmente ser descartado en su edición V (publicada en 2013), donde es reconceptualizado bajo el diagnóstico de "Disforia de género", dejando de ser una enfermedad mental para ser entendido como un malestar clínico relacionado a la condición de género. 
bastante clara, dicha significación política, tal como lo observa Valentina Verbal (2012), lleva a la autodefinición como "travesti" de varios individuos que podríamos entender como transexuales o transgéneros.

Esta última categoría referente al transgenerismo, que completa la triada principal de quienes conforman las llamadas identidades trans, es la de más reciente conceptualización. Muchas veces ha sido considerada como un "punto intermedio" entre los dos conceptos desarrollados anteriormente, pues comparte con la transexualidad la vivencia permanente del género opuesto al asignado según el sexo, pero al igual que el travesti, no pretende necesariamente la intervención quirúrgico-hormonal del cuerpo. Debido a esta ambigüedad, el término transgénero ha llegado a ser calificado como un concepto "paraguas" en la medida en que se hace referencia por medio de él a "todas las personas marginadas $u$ oprimidas debido a su diferencia con o rechazo de las normas tradicionales de género" (Lamas, 2009: 4).

Observamos el surgimiento de un discurso reivindicativo de las identidades trans, que puede considerarse como heredero de las disputas feministas originadas durante la segunda mitad del siglo XX mediante el posicionamiento político que se otorga al género. Según Donna Haraway (1995), la utilización del concepto de género surge de forma más concreta en el lenguaje político-académico junto con el surgimiento de los movimientos de liberación de la mujer tras la Segunda Guerra Mundial. De esta forma, el concepto estaría íntimamente ligado al establecimiento de la teoría feminista, en tanto perspectiva académica como política, además de sus repercusiones en los distintos movimientos sociales de la segunda mitad del siglo XX. En estos orígenes,

(...) las teorías feministas sobre el género tratan de articular la especificidad de la opresión de las mujeres en el contexto de culturas que distinguen entre sexo y género. Esta distinción depende de un sistema relacionado de significados agrupados en torno a una familia de pares binarios: naturaleza/cultura, naturaleza/historia, natural/humano, recurso/producto (Haraway, 1995: 220).

De este modo, la especificidad del concepto de género permitiría analizar la naturalización cultural dada desde la diferencia sexual y que se expresaría en distintos aspectos sociales. A partir de la objetivación del proceso de diferenciación sexual, sería posible desnaturalizar no sólo los órdenes sociales incluidos en la dicotomía hombre/mujer que producen el género, sino también permitiría pensar las formas de exclusión a dicha dicotomía, lo que hace posible posicionar de manera reivindicativa a las identidades trans.

No obstante, los orígenes epistemológicos de las teorías feministas en la segunda mitad del siglo XX serían establecidos en lo que Haraway (1995) denominó como el "paradigma de la identidad de género", el cual "formuló el concepto de identidad genérica dentro del entramado de la distinción entre biología y cultura, de tal manera que el sexo fue relacionado con la biología (...) y el género con la cultura" (Haraway, 1995: 225). Dado esto, buscando evadir lo que todavía observaba como una perspectiva esencialista y dicotómica en los feminismos desarrollados hasta la década de los 80', la filósofa Judith 
Butler buscaría poner en tensión el concepto de género planteando una alternativa que evitase su fragmentación con la noción de sexo.

Para ello, Butler (2007) sostendrá entonces que el género consistiría en una construcción discursiva por la cual sería posible hacer entendibles ciertas prácticas sociales reguladas, las que a su vez darían coherencia a lo que comúnmente se entiende por "identidad sexual/de género". Butler plantearía que "los géneros «inteligibles» son los que de alguna manera instauran y mantienen relaciones de coherencia y continuidad entre sexo, género, práctica sexual y deseo" (Butler, 2007: 72), proponiendo de esta forma una noción sobre género que se aleja de la dicotomía sexo-biología/género-cultura, ampliando así mismo las implicancias del concepto al situarlo en línea a factores relativos al sexo. En lo concreto, la propuesta de Butler implicará "confundir críticamente las categorías de sexo y género (...) y deconstruir la noción de género como substancia/natural"' (Soley-Beltrán, 2009: 35).

Esta sería una de las premisas fundamentales sobre la cual se establecería durante los años 90' la llamada queer theory en el feminismo anglosajón, abriendo un quiebre epistemológico en torno al sujeto político sobre el cual basar una propuesta teórico-política. En esta línea, De Lauretis (2000) propondrá reapropiar el concepto "queer", el cual hacía referencia de manera injuriosa al individuo rechazado y catalogado como "fuera de la norma", y que aplicado al ámbito de la sexualidad resultaba ser un insulto sobre quien incurriera en una transgresión a la concepción tradicional de identidad sexual. Se establece así la posibilidad de reconocer a las identidades trans como sujeto político para una teoría crítica a las políticas sexuales, llevando acabo una "estrategia de subversión performativa" (Córdoba, 2007) que no solo desconoce o invierte el flujo de saberes emanados por la norma binaria, sino que lo hace en términos productivos al reapropiar la fuerza del insulto ahora como fuerza transgresora.

Es necesario notar, no obstante, las dificultades existentes en torno a la traducción no solo del concepto queer ${ }^{4}$, sino que incluso del propio quiebre epistemológico aplicado fuera de los márgenes de la cultura estadounidense: en lo concreto, emulando esta propuesta teórica por medio de la reapropiación de nociones tales como "marica", "tortillera", "trolo", "cola", etc., observamos que no es posible determinar un concepto que recoja las diferentes variantes del español, sobre todo el latinoamericano, de manera tan certera como lo hace el concepto queer en la cultura estadounidense; mientras que, por otra parte, al aplicar sin traducción el concepto queer sobre un contexto cultural ajeno, concluimos en una noción de carácter más bien académico que puede omitir diferencias tales como de raza o socioeconómicas, y por tanto, sería incompleta ${ }^{5}$.

${ }^{4}$ Sobre dicha problemática, conviene revisar el trabajo realizado por Brad Epps (2008) al contrastar el desarrollo de la queer theory en el ambiente anglosajón, frente a los trabajos teóricos desarrollados desde Hispanoamérica.

${ }^{5} \mathrm{Al}$ realizar una reelectura de la obra de Aníbal Quijano, Mara Viveros (2006) supone que cualquier análisis de género y/o sexualidad en América Latina que omita el concepto de raza, es en sí mismo 
Como respuesta a esta problemática, encontramos la propuesta del José Javier Maristany (2008) quien, realizando una relectura de diversos autores latinoamericanos ${ }^{6}$, encuentra en ellos paralelismos a pensamientos que se formularían como base para el posterior desarrollo de la queer theory. Maristany propone lo que denomina como "genealogía diferencial", un dispositivo de análisis según el cual será posible observar un desarrollo teórico y práctico de lo queer en la obra de autores latinoamericanos, aun cuando el término no había sido todavía reapropiado por la academia anglosajona y tampoco dichos autores dieran uso explícito al concepto. Más concretamente, desde la genealogía diferencial de Maristany podemos observar, por ejemplo, el desarrollo teórico latente (y en ocasiones más explícito) presente en la obra del cronista chileno Pedro Lemebel.

Quizás la referencia más concreta en la obra de Lemebel se encuentra en una intervención universitaria llevada a cabo en mayo de 1991 frente a la visita del propio Félix Guattari al país, texto incluido bajo el título "Loco afán" (Lemebel, 2009) en el libro de crónicas del mismo nombre. En él se hace referencia explícita a la necesidad de elaborar una política sexual crítica a partir de la propia experiencia histórica chilena, proponiendo en su obra la figura central de la travesti o "la loca", poniendo en cuestionamiento de forma simultánea el desarrollo del movimiento homosexual que se había expandido desde EE.UU durante la segunda mitad del siglo $X X$, y que a juicio de Lemebel habría adquirido tintes hegemonizantes y colonizadores en contextos extranjeros, particularmente en Latinoamérica.

A juicio de Maristany, la intención de Lemebel es la de dar cabida a las figuras despreciadas en una disputa frente a una nueva hegemonía que coopera en la reestructuración de las políticas sexuales neoconservadoras; y sería aquí donde "podemos señalar un gesto queer pues es sabido que esta perspectiva surge como un espacio de diferencias al especificar orientaciones, corporalidades y prácticas diversas y con una clara postura anti-identitaria frente al sistema birrepresentacional gay/lésbico" (Maristany, 2008: 23). Por ello, en la obra del cronista tendrá tanta importancia el travestismo latino, concretizado para el caso chileno en la figura de "la loca", el cual significaría una manifestación política-corporal de aquel desentendimiento con los movimientos homosexuales de influencia estadounidense, desconfiando de ellos en tanto no fuesen reterritorializados a los contextos y disputas locales con sus historias correspondientes.

Si bien no es posible trazar de manera paralela las implicancias significativas del travesti latino -"la loca" lemebeliana- hacia otras formas de la identidad trans (como el transgénero o el transexual) de manera directa, sí es posible esbozar desde este punto la

incompleto puesto que las construcciones político-sexuales se originan sobre dicho marco de dominación cultural.

6Junto a José Javier Maristany, también Brad Epps (2008) y Paula Arboleda (2011) trabajan sobre la revisión de obras de autores latinoamericanos donde es posible encontrar referencias, aunque no necesariamente explícitas, a fundamentos teóricos de la queer theory. 
Revista Punto Género № 12. Diciembre de 2019

ISSN 0719-0417 / $148-170$

importancia y necesidad de observar el entramado de las políticas sexuales contextuales que determinan las problemáticas trans en nuestro país.

\section{Las identidades trans en el contexto chileno}

Para abocarnos a la situación de las identidades trans en el contexto chileno, podemos comenzar por observar que el primer caso donde se llevó a cabo una Cirugía de Reasignación Sexual en el país ocurrió en la década del 70', aun cuando existieron numerosos casos de intervenciones médicas realizadas a pacientes intersexuales durante buena parte del siglo XX (Barril, 2015), las cuales se encuentran documentadas en distintas revistas médicas del país.

Cabe destacar también que el país es reconocido actualmente como uno de los más avanzados en cuanto a CRS a nivel latinoamericano (Vergara, 2011), al contar con los cirujanos más experimentados de la región. Paradigmático en este punto fue el caso de Marcia Torres, la primera persona en someterse a una CRS en Latinoamérica, en marzo de 1973 en el Hospital San Borja de Arriarán de Santiago, y que se ha constituido como referente tanto para el debate público (sobre todo, por la amplia cobertura de prensa de la época) como también dentro de distintos colectivos de la diversidad sexual ${ }^{7}$.

Como otra forma de ejemplificar este punto, podemos observar los comentarios realizados por la socióloga argentina Anahí Farji Neer (2015) quien, al indagar sobre los debates parlamentarios previos a la aprobación de la LIG en su país, da cuenta de una situación contradictoria: hasta 2010, se exigía a quienes optaran por una readecuación en su sexo legal que contaran con una "genitalidad acorde", vale decir, que se hubiesen sometido a una CRS; no obstante, dicha intervención se encontraba explícitamente prohibida por la legislación argentina desde el año 1967 (con la excepción de contar con un fallo judicial que la permitiese). Es por ello que "la gran mayoría de las personas que solicitaban el cambio registral viajaban previamente a Chile para realizarse dicha intervención" (Farji Neer, 2015: 354), dado a que la experiencia y especialización sobre la intervención por parte de los cirujanos chilenos data, como se ha mencionado anteriormente, desde la década de los 70'.

De esta forma, a pesar de haber contado con una deficiente y hasta despreocupada legislación respecto a esta temática hasta el reciente debate y aprobación de la LIG, existiría un amplio desarrollo en cuanto a la técnica médico-quirúrgica chilena de la transexualidad y la intersexualidad. Estos saberes, no obstante, no pueden extenderse a toda la comunidad trans pues, hasta por lo menos algunos años atrás, no existía un conocimiento acabado por parte del personal hospitalario en torno a los protocolos médicos sobre al trato que debe darse a éstos como pacientes ${ }^{8}$ (Araya et al,

\footnotetext{
${ }^{7}$ Conocida es su referencia para organizaciones de la diversidad sexual, así como también su cercanía con personajes del contexto local tales como Víctor Hugo Robles y Pedro Lemebel. Este último dedica un capítulo en su obra "Zanjón de la Aguada" a relatar sobre la figura de Marcia Torres.

${ }^{8}$ Mientras el 2011 se emite la Circular №34, la cual pretende garantizar el respeto por la identidad de género y nombre social de las personas trans en el sistema de salud público chileno; en el año 2012 se emite la
} 
Revista Punto Género № 12. Diciembre de 2019

ISSN 0719-0417 / 148 - 170

2014), así como también puede notarse una suerte de desinformación respecto a procedimientos médicos rutinarios para su cuidado (Barril, 2015).

Por otra parte, la situación actual de las problemáticas en el contexto chileno que aquejan a la comunidad trans, parece apuntar a una mayor visibilización de las distintas formas en que se expresa la discriminación vivida por dicha comunidad; por lo que bajo el alero del trabajo realizado por diferentes organizaciones en favor de la diversidad sexual, se han hecho patentes durante los últimos años en el contexto nacional las distintas demandas exigidas por la comunidad trans.

\section{Visibilización mediática de la problemática trans}

Previo a la aprobación de la Ley de Identidad de Género a finales de 2018, se observó en distintos medios de prensa un abordaje particular respecto a variados hechos de discriminación y violencia hacia individuos trans, entre los que podemos destacar primero dos casos de homicidio, ambos ocurridos durante enero de 2015 (Fuentes, Méndez y Wood, 2015), y que fueron expuestos por la prensa como crímenes cuya principal motivación se encuentra en la discriminación hacia individuos trans; mientras también se da uso del término "transgenericidio", utilizado por algunas organizaciones de la diversidad sexual.

Si bien se hicieron visibles en el trato dado por la prensa una serie de vaguedades o bien la utilización confusa e imprudente de los términos (referencia a la víctimas sin considerar el proceso de resignificación social en la cual se encontraban, uso indistinto de los conceptos transexual/transgénero/travesti, etc.), es importante destacar que la exposición de éstos casos apunta, tal como lo destacan Fuentes et al (2015: 5), a la búsqueda de una sensibilización similar a la ocurrida en los casos de femicidio, intentando una equivalencia entre ambos sucesos.

Otra forma de ingreso de las problemáticas trans en el trabajo periodístico nacional fue la realización de diversos reportajes por parte de medios televisivos, enfocados en la situación de "niños transgénero". El primero de ellos fue el que llevó a cabo el programa Contacto de Canal 13 (Montenegro y Figueroa, 2015), emitido en mayo de 2015, donde se documenta la historia de Baltazar Escobar, de cinco años, considerado el primer caso en el país donde una familia apoya la transgenericidad de su hijo a través de la prensa. Meses más tarde, se emite en el noticiario 24 Horas de TVN (Pino y Vicencio, 2015) un reportaje sobre tres niños transexuales y las inquietudes expuestas por sus familiares al no contar con una Ley de Identidad de Género en el país. Finalmente, cabe mencionar también la cobertura realizada al documental Niñas azules y niños rosados (2015), emitido en abril de 2016 en el noticiario central de CHV (Pierattini, 2016), donde también se abordaron los casos de dos niños transgénero.

En todos estos reportajes, y a pesar de que se mantiene una imprudente utilización conceptual al momento de hacer referencia a los infantes transgénero, se apela

Circular $N^{\circ} 21$, que viene a corregir la definición otorgada a los individuos trans en el anterior documento, donde se les mencionaba como "afectadas por un trastorno mental".

P U N T O G É N E R O 156 
a una sensibilización de la situación de estos niños y se destaca la ineficacia de la legislación en el país respecto al tema. Por lo demás, al introducir la temática trans por medio de la figura infantil, puede incluso percibirse una suerte de minimización de problemáticas que se han vuelto hasta cotidianas en la vida de adultos trans, cuestión que se agudiza en individuos de estratos sociales más pobres ${ }^{9}$.

Vale mencionar que la mediatización de las problemáticas trans no significa necesariamente el ingreso de las identidades trans en el trabajo periodístico de los medios de comunicación: gran parte las identidades trans han estado presentes bajo una solapa que los estigmatiza, ligada a tópicos considerados como conflictivos en el ámbito social, tales como la delincuencia o el comercio sexual ${ }^{10}$. Ahora bien, al introducirse las problemáticas trans en la agenda mediática, existe un nuevo abordaje y amplitud hacia las identidades trans, cargadas no obstante bajo la representación de sujetos de cuidado y no necesariamente como sujetos de derecho.

Situación distinta es la que se ha logrado notar durante los últimos años en torno a diversas manifestaciones culturales y expresiones artísticas, en particular desde el cine. Si bien podemos recoger experiencias dentro de la contra-cultura local ${ }^{11}$ donde encontramos el posicionamiento de las disidencias como identidades de derecho, es necesario también considerar el caso de la película "Una mujer fantástica" (2017) como punto de inflexión para la visibilización de las identidades trans en el imaginario local desde la producción cultural, así como la relevancia social adquirida por su protagonista Daniela Vega en tanto "referente cultural" de la comunidad trans ${ }^{12}$.

Reconocida es la apertura mediática otorgada a la comunidad trans luego de que la película de Sebastián Lelio recibiese el Oscar a principios de 2018, propiciando el desarrollo de producciones cinematográficas con enfoque sobre las problemáticas trans: como ejemplos, podemos mencionar el documental "En tránsito" (2018) que relata distintas vivencias trans, destacando la figura de la profesora y poeta Mara Rita; o también el trabajo "Claudia tocada por la luna”, centrado en las experiencias de una matrona trans. Aún más, vale mencionar también el ingreso de actores y actrices trans en

\footnotetext{
9 Como ejemplo de esto, podemos evidenciar la nula relación establecida por estos reportajes con otras problemáticas denunciadas históricamente por la comunidad trans, como lo puede ser la precaria situación laboral característica en la que se encuentran los individuos trans en el país. Paralelamente, son estas mismas fuentes periodísticas las que solían reforzar estigmas sobre los individuos trans a lo largo de otras coberturas noticiosas como, por ejemplo, al momento de ligar la figura del travesti con la delincuencia, la violencia y el comercio sexual.

10 Según se mencionaba en la nota anterior, la figura insigne es la del travesti.

${ }^{11}$ Paradigmático es el caso del colectivo "Las Yeguas del Apocalipsis", conformado por Pedro Lemebel y Francisco Casas, surgido hacia finales de los 80' y que a través de sus performances buscó dar relato a distintas problemáticas y estigmas sociales que aquejaban a la comunidad disidente de la época, como lo fue la expansión del sida en el país (Contardo, 2011: 341-342). Más contemporáneo es el trabajo de visibilización llevado a cabo por el Colectivo Universitario de Disidencia Sexual (CUDS), cuyas intervenciones en favor de la despenalización del aborto les llevaron a recibir una acusación por "asociación ilícita" en 2012 (CUDS, 2013).

12 Importante es notar las reflexiones de Andrés Ibarra (2019: 29) a propósito de las distancias que se hallan entre la figura de "la loca lemebeliana" y el personaje de Marina, interpretado por Daniela Vega en el filme.
}

P U N T O G É N E R O 157 
largometrajes cuyo enfoque no es precisamente dar cuenta de la vivencia trans, como es el caso de Demian Hernández en la película "Tarde para morir joven" (2019), interpretando al personaje de Sofía.

\section{Derechos Humanos y la comunidad trans en el país}

Junto a esta visibilización mediática, las problemáticas trans también tuvieron lugar en distintas publicaciones de estudios que analizaban la situación de los Derechos Humanos en el país, entre los cuales podemos considerar las realizadas por el Centro de Derechos Humanos de la Universidad Diego Portales, en sus Informes Anuales de 2013 y 2015. Para el informe realizado el año 2013, por primera vez se da especial cabida a la situación de la población trans en el ámbito local en el artículo dedicado anualmente para el contexto de la diversidad sexual.

En dicha oportunidad, se compara la situación del debate en torno a los Derechos Humanos de los individuos trans a nivel internacional con respecto a la realidad chilena, y posteriormente se realiza una síntesis del proyecto de Ley de Identidad de Género ingresado ese año al Congreso. El artículo observaba que "estas omisiones de los deberes de respetar, proteger y garantizar los derechos de las personas LGTBI se muestran en toda su gravedad en el caso de las personas trans" (Vial, 2013), caso que se plantea como una deslegitimación tanto identitaria como corporal.

Por su parte, estas problemáticas vuelven a ser tratadas con profundidad en el Informe realizado en el año 2015, donde en primera instancia se observó el progreso que había tenido la tramitación de la LIG, así como los discursos que surgieron en oposición a la misma; para posteriormente analizar la "evolución jurisprudencial en causas sobre cambio de nombre y sexo registral" (Álvarez, del Pino y Vial, 2015: 382), donde se calificó como favorable el "cambio de paradigma" ocurrido en casos donde los jueces apuntaban cada vez menos a la exigencia de intervención quirúrgica para el reconocimiento legal del cambio de nombre y sexo en individuos trans. No obstante, se objetaba que esto seguía ocurriendo a un nivel particular y a criterio de cada juez, al no encontrarse en aquel entonces una regulación jurídica.

Finalmente, en un documento realizado en 2014 por el Instituto Nacional de Derechos Humanos, se analizan las problemáticas de las identidades trans en el país, sobre las cuales se afirma que "la identidad de las personas trans es independiente a las cirugías o tratamientos a los cuales pueden optar, no pudiendo exigirse ningún tipo de intervención para un efectivo reconocimiento de su identidad de género" (INDH, 2014: 19), haciendo eco de los tratados internacionales respecto a los Derechos Humanos de la comunidad trans, y apoyando la tramitación expedita de la LIG.

Vemos que, ligado a la observación de los derechos de la diversidad sexual, las problemáticas trans tienen su cabida en los estudios sobre Derechos Humanos en el país al menos durante los 5 años previos a la aprobación de la LIG. Los estudios realizados por distintas entidades fijaban principalmente su atención sobre el ambiente de desprotección a propósito de las identidades trans desde el margen legal, vale decir, 
aluden a que los diferentes hechos de violencia (física, psicológica, laboral, médica, judicial, etc.) hacia personas trans debían ser abordados precisamente a través de la promulgación de una LIG.

Estas indagaciones, al igual que las revisadas en el contexto de la diversidad sexual, son puestas en relación comparativa con la situación en otros países tanto de la región como de Europa o en Estados Unidos. De manera especial, se toma como referencia el desarrollo de discusiones y promulgaciones de leyes de identidad de género en distintos países de América Latina durante el último tiempo. Respecto a esto, la Ley aprobada en Argentina en el año 2012 sirvió como referencia de estudio para el caso de los Derechos Humanos de personas trans en el país, entendiendo el caso argentino como un ejemplo de puesta en práctica de una Ley que hasta la fecha parecía muy lejana y propia de otro tipo de sociedades pensadas como "más desarrolladas".

\section{Surgimiento y consolidación de organizaciones sociales}

En conjunto con el trabajo realizado por investigaciones de los Derechos Humanos en torno a la situación de la comunidad trans en el país, durante los últimos años se observó un creciente surgimiento y consolidación de distintas organizaciones que intentan abordar las problemáticas de personas transexuales, transgéneros, travestis e intersexuales en el contexto nacional de forma específica. Entre las varias organizaciones de apoyo a las identidades trans, podemos encontrar las ramas concretas de las asociaciones por la diversidad sexual más influyentes, como el MUMS Chile, la Fundación Iguales y el Movilh, así como también se encuentran organizaciones específicamente trans como lo son la asociación Organizando Trans Diversidades (OTD Chile, con participación política y comunitaria de personas trans), el Sindicato Amanda Jofré (conformado por trabajadoras sexuales trans) y fundaciones como Selenna o TranSítar (ambas ligadas al cuidado y defensa de la niñez trans), varias de ellas llegando a estar agrupadas en el "Frente de la Diversidad Sexual" como red de trabajo conjunto de la comunidad LGTBI en el país, y que a la fecha funciona como espacio de difusión para sus labores.

Por medio del trabajo realizado por estas organizaciones, se buscó el establecimiento concreto y mayor visibilidad de las problemáticas trans en el contexto nacional, y la piedra angular parecía encontrarse en la exigencia de una LIG. A través de ésta se esperaba la regularización de la ambivalencia en torno al reconocimiento legal de las identidades trans; por otro lado, también se pretendía sentar las bases para abordar la inclusión de sujetos trans en el ámbito del trabajo, mediante la búsqueda de la resolución del denominado "círculo de problemas laborales trans"13; finalmente, otro punto al interior

\footnotetext{
13 Valentina Verbal (2011) define el "círculo de problemas laborales trans" de la siguiente forma: "consiste en que para trabajar, el documento nacional de identidad debe concordar con la apariencia externa; para modificar este documento, hay que someterse a una cirugía de reasignación sexual; para acceder a esta cirugía, hay que disponer de recursos; y para tener recursos, hay que trabajar" (2011: 16).
} 
de las problemáticas que se pretendían resolver trataba sobre la correcta jurisdicción en torno a los casos de discriminación por identidad de género, sobre lo cual no existían precedentes en el país de un fallo favorable para individuos trans (PETRA y Fundación TranSítar, 2016).

Las demandas para la tramitación de una LIG en el país, decantadas en su promulgación a finales de 2018, se manifestaron en paralelo a otras exigencias provenientes principalmente de organizaciones feministas y de la disidencia sexual, varias de ellas de carácter histórico y que se concretaron en hitos importantes durante la última década, tales como las promulgaciones de la Ley Antidiscriminación en 2012, el Acuerdo de Unión Civil en 2015, y la Despenalización del Aborto en tres causales en 2017. Estas disputas pueden observarse como preámbulo para el estallido de manifestaciones sociales ocurridas durante el llamado "Mayo Feminista" en 2018, propiciadas en gran medida por una serie de denuncias contra casos de acoso, abuso y violencia contra la mujer, emanadas sobre todo desde sectores estudiantiles (Reyes-Housholder y Roque, 2019: 196). Esta serie de manifestaciones extendidas durante gran parte del año, marcaron pauta tanto en materia gubernamental como en labores legislativas, así como también es posible destacar en ellas la reconocida filiación entre las variadas demandas feministas con las de la diversidad y disidencia sexual (Reyes-Housholder y Roque, 2019): la posibilidad de utilizar el nombre social en la documentación universitaria para el caso de personas transgénero, la elección de una presidenta trans en la Federación de Estudiantes de la Universidad de Chile, y la propia tramitación de la LIG a finales del mismo año son ejemplo de esto.

Debido a su participación en la elaboración del proyecto de Ley de Identidad de Género, OTD Chile se configuró como una de las principales organizaciones que abordaban (y abordan hoy en día) las problemáticas trans en el contexto nacional de manera específica, presentando un trabajo con más de 10 años de experiencia, con presencia tanto en el ámbito legislativo como el comunitario.

Esto último da cuenta de que, si bien a propósito de las disputas llevadas a cabo por la promulgación de una Ley de Identidad de Género en el país existe un reciente y creciente conocimiento del trabajo llevado a cabo por distintas organizaciones sociales, muchas de éstas llevan ya varios años de trabajo de carácter comunitario, referido a prestar apoyo al contexto problemático y de estigma social en el que viven personas trans. Junto al caso de OTD Chile recién comentado, podemos también mencionar el trabajo desarrollado desde el año 2004 por el Sindicato Amanda Jofré, presentado como un "sindicato independiente de trabajadoras sexuales travestis, transgéneros y otras"14; 0 bien el surgimiento a principios de la década del 2000 de Traves Chile, primera agrupación de personas trans que adquiere estatus jurídico-legal en el país (Robles, 2008).

Junto a la visibilización mediática del trabajo realizado por diferentes entidades de trayectoria dentro de la comunidad trans en el país, tuvieron también especial surgimiento

\footnotetext{
${ }^{14}$ http://www.amandajofre.cl/historia.html
} 
a propósito del debate por la Ley de Identidad de Género distintas agrupaciones dedicadas de manera más exclusiva y particular a las problemáticas relativas a la niñez trans. En este caso, destacan nombres como la Fundación TranSítar, la Fundación Selenna, o bien la Fundación Todo Mejora (esta última con principal enfoque en el ambiente escolar, abordando problemáticas como el bullyng en colegios hacia toda discriminación sufrida ya sea por orientación sexual o de género).

\section{Identidades y problemáticas trans en el contexto académico}

El proceso de visibilización del cual han sido objeto las problemáticas trans está vinculado también al reciente interés por su estudio en diferentes ramas de las ciencias sociales en el país. Respecto a esto, Pilar Errázuriz señala que, si bien el desarrollo del feminismo durante la dictadura tuvo su repercusión académica en el surgimiento de distintos centros de estudios de género, existiendo una gran fluidez de diálogo entre ambos, por otra parte

“(...) los estudios de la academia nuestra han ido evolucionando a lo largo de los últimos diez años, no sé si tanto con los movimientos sociales con respecto a la diversidad, sino en cuanto al fenómeno perfectamente legítimo y discernible de todo lo que está sucediendo ahora con los colectivos gays, lésbicos y trans, etc." (Yáñez, 2015: 103)

En otras palabras, el interés por el estudio de las identidades trans, a diferencia de lo ocurrido con la relación entre los movimientos feministas y los estudios de género, surge en la academia chilena de manera más tardía que el propio trabajo (sobre todo comunitario) que vienen realizando distintas organizaciones por la diversidad sexual, viniendo a observar primero los efectos y resultados de su labor en la sociedad. Esto deriva en un extendido predominio de enfoques biologicistas para atender la situación de las identidades trans en cuanto a estudios académicos.

Junto con la apertura del debate en el marco legal, comienzan a estudiarse los fenómenos relacionados a las identidades trans en el contexto nacional, pero siempre ligados a los estudios de género (que se encuentran ya establecidos e institucionalizados) como a los de la homosexualidad. Ejemplo de esto lo encontramos en el trabajo realizado por Maritza Carrasco (2002), donde travestis y transexuales son incluidos en lo que la autora denomina como subcultura homosexual, entendiendo esto último como un "mundo oculto" que se encuentra omitido al interior de la sociedad chilena.

La homologación de dichas identidades trans a la población homosexual es argumentada por Carrasco comprendiendo que, en tanto "las categorías sociales homosexuales, en particular la categoría gay, constituyen el "grupo mayoritario» y más conocido socialmente" (2002: 14), tanto travestis como transexuales se "iniciarían" ingresando primero al circuito homosexual.

Posteriormente, se dará espacio para el estudio de los sujetos trans en otras disciplinas ajenas a las ciencias médicas, especialmente en la psicología y el derecho. 
Para el caso de la psicología, surgen una serie de trabajos referentes a las construcciones identitarias de las cuales son objeto sujetos transexuales, transgénero y travesti, mediante la realización de estudios de casos.Entre dichos estudios, podemos destacar los realizados por Ormeño (2007), Cea (2008) y Fuentes et al (2015), los cuales mantienen como reflexión final el describir el proceso de "volverse trans" como una cuestión que, si bien permite una experimentación y expresión plena del sí mismo (Cea, 2008), implica también deslegitimación social y relacional, que se ve expresada en diversos ámbitos, tales como en el ambiente laboral, la relación con familiares y amistades, el quehacer cotidiano, etc.

También se puede destacar que en las tres investigaciones mencionadas, el cuerpo ocupa un lugar fundamental en el análisis de construcción identitaria, ya sea que se entienda como negado en tanto referente biológico por parte de los sujetos trans (Ormeño, 2007), o porque constituye un referente que puede ser modificado y moldeado para la expresión verdadera de la identidad (Cea, 2008). Junto a estas investigaciones, se han publicado recientemente distintos artículos que abordan la cuestión de la construcción identitaria en individuos trans, entre los que cabe mencionar los artículos realizados por Altamirano et al (2012) y Noseda (2012), los cuales coinciden en reconocer que existen diferencias entre la construcción identitaria de transexuales femeninos y masculinos.

Los estudios realizados desde el derecho son más recientes, y apuntan principalmente a analizar la ambigua situación jurídico/legal en la que se encontraban los sujetos trans previo a la aprobación de la Ley de Identidad de Género. Podemos rescatar, por un lado, el estudio realizado por Fernanda Gajardo (2014) donde analizan las circunstancias en las que se encuentran infantes y adolescentes trans y homosexuales internados en centros del SENAME; como también la investigación de Pacheco y Silva (2015) acerca de la situación del cambio del nombre en individuos trans como posibilidad de resignificación sexual en términos legales. Esta última problemática muestra un gran interés para la disciplina, pues aparece también trabajada en distintos artículos acerca de los Derechos Humanos (cuestión revisada anteriormente), así como también representa, a juicio de Muñoz León (2015) un punto de "complementariedad epistemológica" entre la medicina y el derecho.

Este desarrollo de las temáticas trans en las ciencias sociales, es coherente con el asentamiento que han tenido las problemáticas de la diversidad sexual como materia de estudio en la academia chilena; que aun no habiendo alcanzado altos niveles de consolidación e institucionalización, pueden ser ejemplificadas más concretamente en las ediciones recientes de revistas científicas que han dedicado números especiales al trabajo realizado desde nuevas propuestas teóricas en torno a los estudios de género ${ }^{15}$

\footnotetext{
${ }^{15}$ Como referencia, véase la edición N4 de la revista Punto Género (2014), del Núcleo de Género y Sociedad Julieta Kirkwood de la Universidad de Chile.
} 
que acogen estas problemáticas, $u$ otras ediciones ${ }^{16}$ que reúnen estudios cuyo principal enfoque es el contexto de la diversidad sexual en el país.

De manera general, se puede observar que el asentamiento de las problemáticas trans dentro del trabajo académico en el país mantiene dos puntos de arraigo. Por un lado, su entrada va ligada primero hacia los estudios de la diversidad sexual, con orígenes a finales de la década de los 90' y con mayor asentamiento hacia la década del 2000; entrada que en todo caso se da de manera mucho más temprana al menos de forma específica. A su vez, los estudios tanto de la diversidad sexual como de las problemáticas trans se encuentran supeditados a la profundización de estas temáticas siempre al alero de los Estudios de Género, los cuales como se ha dicho, se encuentran desenvueltos e institucionalizados por lo menos desde mediados de los 90'. Esta especificación temporal cobra importancia justamente por lo mencionado anteriormente, respecto a que la entrada de las problemáticas trans en la academia chilena se observa gracias al desarrollo y proliferación del trabajo de las distintas organizaciones sociales en el país. En concreto, esto implica que no fue necesario un desarrollo teórico, específico y concreto de nuestro contexto nacional, para el surgimiento de este tipo de organizaciones; más bien, dichos planteamientos teóricos vienen a hablar a partir de las implicaciones en el trabajo comunitario, intentando primero desapegarse de los enfoques biologicistas que conservan aún gran arraigo tanto dentro de la academia como en el imaginario colectivo.

\section{Importancia del cuerpo en las problemáticas trans}

Revisados estos antecedentes, podemos centrarnos ahora en el lugar que ocupa el cuerpo respecto a las problemáticas trazadas en torno a los individuos trans en el contexto nacional, cuestión que parece mostrarse transversalmente en cuanto las ciencias sociales han abordado el caso de las problemáticas de la diversidad sexual y, en específico, de los sujetos trans. Analizando lo que implican socialmente las disposiciones corporales en el Chile de post-dictadura, Pincheira (2009) supone la existencia de un cierto "orden corporal" que, articulado en las relaciones sociales, exigiría ciertas formas de control político sobre los cuerpos; cuestión que parece hacerse visible en el caso de los individuos trans y en el contexto de problemáticas en el cual se encuentran. De esta forma, podemos encontrar también estudios más bien propositivos respecto a la resolución de las problemáticas trans en el contexto nacional y que no se centran solo en la denuncia, como la idea trabajada por Martín Torres (2011) quien supone la transexualidad como una reapropiación del cuerpo pensando éste como un territorio que se convierte en una "arena de batalla".

Se observa entonces que la situación de los cuerpos trans tiende a ser pensada en permanente conflicto, cuestión que se advierte en las propias fuentes originarias que

\footnotetext{
${ }^{16}$ La edición $N^{\circ} 7$ de la revista Liminales (2015), de la Escuela de Psicología de la Universidad Central, se presenta como la primera edición de una revista universitaria del país donde se abordan exclusivamente las temáticas de género y diversidad sexual, participando en ella académicos y activistas de la comunidad LGTBI.
} 
delimitan las identidades trans, a saber, en su relación con el campo médico. Respecto a esto, en un estudio sobre la percepción de transexuales acerca del sistema de salud chileno, Araya et al (2014) observan que la principal falencia radica en la falta de un conocimiento acabado de los protocolos en las instituciones médicas del país para el trato de éstos como pacientes; hecho que es avalado por Barril (2015) en cuanto afirma un desconocimiento por parte del personal médico sobre el modo de tratar a pacientes trans:

(...) la ignorancia de los mismos profesionales inhibe la posibilidad de dar un trato adecuado para la población trans, ya que la mayoría de los médicos no sabría cómo atenderlos (...) lo que indica que el procedimiento habitual es la derivación hacia especialidades médicas más acordes al fenómeno (2015: 22).

Dicho descuido se traduce en una hegemonía de manuales psiquiátricos para el tratamiento de pacientes trans, lo que a su vez significa su constante patologización. Esta situación de desconocimiento de los individuos trans por parte del discurso médico en el país, entra en contradicción en cuanto tiende a suponerse éste como la principal autoridad para pensar los cuerpos trans, y todo cuerpo sexuado, a nivel social (Martínez, M., 2005: 114), y sobre el cual se sientan las bases biologicistas que establecen políticas de ordenamiento corporal en el país (Pincheira, 2009: 95).

No obstante, tal como afirma el mismo Pincheira (2009: 101), será por medio del significado social adquirido por los cuerpos que se reafirmarán las disposiciones construidas en torno al orden corporal; dado que el control social estaría asegurado en tanto que le otorga a un cierto cuerpo un determinado valor simbólico. Por lo tanto, cabe pensar entonces cómo es significado el cuerpo trans en la sociedad chilena, entendiendo que se constituye como un proceso de producción social del propio sexo de los individuos trans, pero que también se corresponde con los márgenes en los cuales se enmarca la producción del sexo en la sociedad en general. El cuerpo se establece como un medio que, al adquirir ciertas significancias sexuales, adquiere sentido en términos sociales; lo cual para el caso de individuos trans, si bien tiende a manifestarse de manera estigmatizante, parece transformarse para los colectivos por los derechos de la diversidad como un referente político de reivindicación, cuestión que puede verse ejemplificada en la disputa llevada a cabo por la Ley de Identidad de Género.

A su vez, dicha exigencia puede entenderse como una búsqueda por "legalizar" el cuerpo trans, lo cual intenta entonces disputar sus orígenes médico-patologizantes bajo la idea de que la identidad de género debe ser reconocida sin la necesidad de intervenciones quirúrgicas previas. Así, el interés se detiene en la relación que tienen los individuos trans con sus propios cuerpos y la (re)significación social que buscan otorgarles. Respecto a esto, el caso de los individuos trans se vuelve paradigmático para el estudio de los mecanismos sociales por medio de los cuales se construye el sexo a través de la significación del cuerpo, en tanto éstos serían más conscientes de la utilización de sus propios cuerpos como "artefactos" (Soley-Beltrán, 2009: 22). Así mismo, podemos suponer que en el caso de individuos trans sea también posible observar dicho proceso de significación en atención con la situación conflictiva en la cual se encuentra la 
Revista Punto Género № 12. Diciembre de 2019

ISSN 0719-0417 / 148 - 170

comunidad trans en el país, en tanto intuimos que éstos se hayan mayormente enterados de las distintas problemáticas expuestas anteriormente (aun después incluso de la aprobación de la Ley de Identidad de Género).

\section{Conclusiones}

Durante el presente trabajo, se ha realizado un recorrido por distintos tópicos a través de los cuales se han introducido las problemáticas trans en el debate público del contexto chileno. Se ha observado primero el cambio de paradigma acaecido en los últimos años a través de los medios de comunicación, donde si bien se ha diluido la figura estigmatizada de las identidades trans, se ha sustituido ésta por una apariencia victimizante (similar al caso ocurrido por la figura de la mujer frente al creciente abordaje de la violencia de género); vale decir, como una figura de resguardo y cuidado antes que de derechos.

Posteriormente, se verificó la persistente inclusión de las problemáticas trans a través de diversos estudios sobre la situación de los Derechos Humanos en el país, persistencia que emerge de manera paralela con la puesta en debate por una Ley de Identidad de Género en el Congreso, y que se encuentra altamente mediada por la relación comparativa realizada a propósito de la situación en el extranjero, tanto a nivel latinoamericano como con el continente europeo. Análogamente, se observa la consolidación de diferentes organizaciones que se abocan a las problemáticas trans, varias de ellas con un amplio trabajo comunitario, pero también con algún eco en organizaciones de trayectoria dentro de las disputas por la diversidad sexual. Paralelamente, se observa un importante cambio de paradigma desde la producción cultural del país (sobre todo desde el cine), así como su filiación a disputas no solo de organizaciones de la diversidad sexual sino también los movimientos feministas.

Se analizó también la paulatina entrada de las problemáticas trans en el contexto académico local, fuertemente ligado al desarrollo de investigaciones en torno a la diversidad sexual, y que tienen su base en los Estudios de Género ya institucionalizados desde la década de los 90'. Se releva que, a diferencia de éstos últimos que obtienen su consolidación gracias a la fuerte influencia de la teoría feminista establecida décadas antes, los estudios a propósito de la diversidad sexual y de las problemáticas trans en particular vienen a responder al desarrollo del trabajo comunitario expresado a través de distintas organizaciones sociales.

El punto en común entre todos estos tópicos refiere a la problematización del cuerpo y sus concepciones esencialistas como lugar de referencia. Suponer la entrada de las problemáticas trans, a través de los distintos tópicos revisados, implica necesariamente revisar las formas en que los preceptos esencialistas del cuerpo se encuentran normalizados en nuestra sociedad, permitiendo entonces poner al cuerpo (y sus distintas referencias a través, por ejemplodel sexo o el género) en el centro del debate público. 
El presente bosquejo pretende entonces relevar la importancia del cuerpo a propósito de este debate, presentando un interés acerca de sus distintos procesos de significación y (re)significación. A raíz de esto, toman fuerza ideas como las de Martín Torres (2011) al observar el cuerpo como "arena de batalla", ya que la visibilización de las problemáticas trans supone necesariamente una puesta en debate acerca de los preceptos tradicionales en torno a las distintas formas de significancia del cuerpo.

\section{Bibliografía}

Álvarez, Juan José; del Pino, Sebastián y Vial, Tomás (2015): "Derechos de la diversidad sexual 2015: avances y falencias", en Vial, Tomás (ed.): Informe Anual Sobre Derechos Humanos en Chile 2015; Ediciones Universidad Diego Portales, Santiago.

Altamirano, Jonny; Araya, Claudia; Arias, Francisca; Ruiz, Loreto y Orellana, Zicri (2012): "Identidad transexual: un proceso de género en tránsito"; en Revista Pequén, Vol. 2, № 1, Universidad del Bío-Bío, Concepción, pp. 108-124. Disponible en: http://www.ubiobio.cl/pequen/

Araya, Constanza; Carreño, Rocío; Constanzo, Josette; Contreras, Claudia; Manosalva, Rocío y Vielma, Tomás (2014). "Percepción de los/las transexuales sobre el trato entregado por el sistema de salud chileno privado y público"; en Revista de Estudios Cualitativos REC, Vol. 1, N 1, USACH, Santiago, pp. 63-80.

Arboleda, Paola (2011): “¿Ser o estar «queer» en Latinoamérica? El devenir emancipador en: Lemebel, Perlongher y Arenas", en Íconos. Revista de Ciencias Sociales; № 39, Facultad Latinoamericana de Ciencias Sociales - Sede Académica de Ecuador, Quito, p. 111-121.

Barril, Juan Pablo (2015): "La (re)asignación sexual en Chile: la (re)territorialización de la ambigüedad del género por el discurso médico del sexo binario"[Seminario de grado]; Universidad Alberto Hurtado, Santiago.

Benjamin, Harry (1999): The transsexual phenomenom [Edición electrónica];Symposium Publishing, Düsseldorf.

Butler, Judith (2007): El género en disputa. El feminismo y la subversión de la identidad. Ediciones Paidós, Barcelona.

Carrasco, Maritza (2002): "Subculturas homosexuales. Mundo Oculto de gays, travestis y transexuales" [Tesis de grado]: Escuela de Sociología, Universidad de Chile, Santiago. 
Revista Punto Género № 12. Diciembre de 2019

ISSN 0719-0417 / 148 - 170

Cea, Sandra (2008): "Estudio cualitativo de las representaciones de género femenino en la construcción identitaria de un sujeto travesti femenino" [Tesis de grado]; Escuela de Psicología, Universidad de Chile, Santiago.

Contardo, Óscar (2011): Raro. Una historia gay de Chile, Cap.7 "La dictadura: pasos de baile"; Editorial Planeta Chilena S.A., Santiago.

Córdoba, David (2007): "Teoría queer: Reflexiones sobre sexo, sexualidad e identidad. Hacia una politización de la sexualidad", en Córdoba, David; Sáez, Javier y Vidarte, Paco (comps.): Teoría Queer. Políticas Bolleras, Maricas, Trans, Mestizas; Editorial Egales, Madrid.

Colectivo Universitario de Disidencia Sexual (2013): "Asociación Ilícita", Transcripción completa de denuncia de asociación ilicita contra la CUDS por la Campaña "Dona por un aborto ilegal"; Cuaderno de Disidencia Sexual, Tomo 1, Ediciones Sexuales Editores, Santiago.

de Lauretis, Teresa (2000): “La tecnología del género", en Diferencias. Etapas de un camino a través del feminismo; Editorial horas y Horas, Madrid.

del Pino, Sebastián y Verbal, Valentina (2015): "El proyecto de ley de identidad de género. Una aproximación a sus disposiciones y fundamentos"; en Liminales, Año 4, $\mathrm{N}^{\circ} 7$, Universidad Central de Chile, Santiago, pp. 180-202. Disponible en: http://www.ucentral.cl/revistas-escuela-de-psicologia/prontus ucentral2012/2012-11$\underline{\text { 22/103041.html }}$

Epps, Brad (2008): "Retos, riesgos, pautas y promesas de la teoría queer", en Revista Iberoamericana, Vol. LXXIV, № 225, University of Pittsburgh, Pittsburgh, pp. 897-920.

Farji Neer, Anahí (2015): "Cuerpo, derechos y salud integral: Análisis de los debates parlamentarios de las leyes de Identidad de Género y Fertilización Asistida (Argentina, 2011-2013)"; en Salud Colectiva, Año 3, N 11, Universidad de Lanús, pp. 351-365.

Fernández, Josefina (2004): Cuerpos desobedientes. Travestismo e identidad de género; Editorial Edhasa, Buenos Aires.

Foucault, Michel (1987): Historia de la sexualidad 1. La voluntad de saber; Siglo XXI Editores, México D.F.

Fuentes, Macarena; Méndez, Felipe y Wood, Luis (2015): "Agenciamientos del devenir transexual en el Chile contemporáneo. Una historia de vida" [Tesis de grado]; Escuela Psicología, Universidad Academia de Humanismo Cristiano, Santiago. 
Gajardo, Fernanda (2014): "Niños, niñas y adolescentes lesbianas gays bisexuales trans privados de libertad: vulneración de derechos, acciones para su protección" [Tesis de grado]; Facultad de Derecho, Universidad Alberto Hurtado, Santiago.

Haraway, Donna (1995): Ciencia, cyborgs y mujeres. La reinvención de la naturaleza, Cap. 5 "«Género» para un diccionario marxista: la política sexual de una palabra"; Ediciones Cátedra, Madrid.

Ibarra, Andrés (2019): "El cuerpo transgénero en Una mujer fantástica"; en Badebec, Vol. 9, №17, Facultad de Humanidades y Artes, Universidad Nacional de Rosario, pp. 22-45.

INDH, (2014): "Informe Proyecto de ley que reconoce y da protección al derecho a la identidad de género"; Boletín 8924 - 07, Instituto Nacional de Derechos Humanos. Disponible

en:http://bibliotecadigital.indh.cl/bitstream/handle/123456789/648/Informe.pdf[Fecha de consulta: 11 de mayo, 2016].

King, Dave (1998): "Confusiones de género: concepciones psicológicas y psiquiátricas sobre el travestismo y la transexualidad”, en Nieto, José Antonio (comp.): Transexualidad, transgenerismo y cultura. Antropología, identidad y género; Editorial Talasa, Madrid.

Lamas, Marta (2009): “El fenómeno trans”; enDebate Feminista, Año 20, Vol. 39, México D.F., pp. 3-13.

Lemebel, Pedro (2009): Loco Afán. Crónicas de sidario; Seix Barral, Santiago

Maristany, José Javier (2008): “¿Una teoría queer latinoamericana?: Postestructuralismo y políticas de la identidad en Lemebel”; en Lectures du genre, $\mathrm{N}^{\circ} 4$ : Lecturas queer desde el Cono Sur, pp. 17-25.

Montenegro, Paz (real.) y Figueroa, Paulina (inv.) (2015, 17 de mayo): "Mi hija es transgénero", en Cáceres, Camila (prod.): Contacto [programa de televisión];Canal 13, temporada 2015 cap. 1, Santiago. Disponible en: http://www.13.cl/programas/contactot25/capitulos/mi-hija-es-transgenero [Fecha de consulta: 9 de mayo, 2016].

Muñoz León, Fernando (2015): "El reconocimiento legal de la transexualidad en Chile mediante el procedimiento judicial de cambio de nombre. Un caso de complementariedad epistemológica entre medicina y derecho"; en Revista Médica de Chile, Vol. 143, № 8 , Santiago, pp. 1015-1019.

Noseda, Janet (2012): "Muchas formas de transexualidad: diferencias de ser mujer 
Revista Punto Género № 12. Diciembre de 2019

ISSN 0719-0417 / 148 - 170

transexual y de ser mujer transgénero"; en Revista de Psicología, Vol. 21, $\mathrm{N}^{\circ} 2$, Universidad de Chile, Santiago, pp. 7-30. Disponible en: http://www.facso.uchile.cl/publicaciones/87922/vol-21-n-2-2012-de-la-revista-de-psicologia

Ormeño, Myriam (2007): "Una aproximación a la construcción de identidad de las personas denominadas «trans», a través de las narrativas de sus experiencias vivenciales articuladas con la participación en organizaciones relacionadas a la disforia de género, que se encuentran ubicadas en la Región Metropolitana y Quinta Región" [Tesis de grado]; Escuela de Psicología, Universidad de Humanismo Cristiano, Santiago.

Pacheco, Jorge y Silva, Eduardo (2015): "Análisis de la legislación, procedimiento y jurisprudencia de las rectificaciones de partida de nacimiento por cambio de nombre y sexo de personas transexuales" [Tesis de grado]; Facultad de Derecho, Universidad de Chile, Santiago.

PETRA y Fundación TranSítar (2016, 10 de mayo): “Demandan a pub «El Túnel» por discriminación contra mujer trans";Closet [revista digital], Santiago. Disponible en: http://www.revistacloset.cl/index.php/2016/05/10/demandan-a-pub-el-tunel-pordiscriminacion-contra-mujer-trans/ [Fecha de consulta: 11 de mayo, 2016].

Pierattini, Daniella (2016, 25 de abril): "La difícil realidad de los niños transgénero en Chile”, en Chaldichoury, Patricio (dir.): Chilevisión Noticias [noticiero]; CHV, Santiago. Disponible en: http://www.chilevision.cl/noticias/chvnoticias/reportaje-a-fondo/la-dificilrealidad-de-los-ninos-transgenero-en-chile/2016-04-25/120153.htm[Fecha de consulta: 9 de mayo, 2016].

Pincheira, Iván (2009): "Las políticas del cuerpo en el Chile de las post-dictadura: entre el (bio)poder y la resistencia"; en Sociedad Hoy, № 16, Universidad de Concepción, Concepción, pp. 93-105. Disponible en: https://www.sociedadhoy.cl/

Pino, Ivonne y Vicencio, Felipe (2015, 4 de agosto): "Niños transexuales: la lucha por la identidad en Chile", en Luengo, Alberto (dir.): 24 Horas [noticiario]; TVN, Santiago. Disponible en: http://www.24horas.cl/nacional/ninos-transexuales-la-lucha-por-laidentidad-en-chile-1745865 [Fecha de consulta: 9 de mayo, 2016].

Reyes-Housholder, Catherine y Roque Beatriz (2019): "Chile 2018: desafíos al poder de género desde la calle hasta La Moneda”; en Revista de Ciencia Política, Vol. 39, № 2, Santiago, pp. 191-215.

Robles, Víctor Hugo (2008): Bandera Hueca. Historia del Movimiento Homosexual en Chile; Editorial Cuarto Propio, Santiago. 
Revista Punto Género № 12. Diciembre de 2019

ISSN 0719-0417/ $148-170$

Soley-Beltrán, Patricia (2009): Transexualidad y la matriz heterosexual. Un estudio crítico de Judith Butler, Edicions Bellaterra, Barcelona.

Torres, Martín (2011): "Territorialización del cuerpo: el cuerpo como un espacio de lucha"; en Revista Geográfica de América Central, № Especial EGAL, Costa Rica, pp. 1-11.

Vergara, Carlos (2011, 18 de septiembre): "La paradoja de Chile: líder en cirugías, pero no acepta el matrimonio gay"; La Nación, Buenos Aires, p. 24.

Verbal, Valentina (2011, 28 de julio): "Hey, soy transexual y también existo"; The Clinic, Año 12-N404, Santiago, p. 16.

Verbal, Valentina (2012): "Transexualidad en Chile: Derechos Humanos y desafíos políticos"; Fundación Iguales, Valparaíso.

Vial, Tomás (2013): "Informe sobre diversidad sexual: las violaciones a los derechos humanos de las personas trans en Chile", en Vial, Tomás (ed.): Informe Anual Sobre Derechos Humanos en Chile 2013; Ediciones Universidad Diego Portales, Santiago.

Viveros, Mara (2006): "Introducción: ¿Qué significa hablar sobre género y sexualidad en América Latina?”, en Viveros, Mara; Rivera, Claudio y Rodríguez, Manuel (comps.): De mujeres, hombres y otras ficciones...Género y sexualidad en América Latina; Tercer Mundo, Universidad Nacional de Colombia, Bogotá.

Yáñez, Ignacio (2015): "Perspectiva de Género en el Siglo XXI. Entrevista a Pilar Errázuriz Vial”; en Revista Liminales, Año 4, № 7, Universidad Central de Chile, Santiago, pp. 100110. Disponible en: http://www.ucentral.cl/revistas-escuela-depsicologia/prontus ucentral2012/2012-11-22/103041.html 